\title{
Contraction of cold neutron star due to in the presence a quark core
}

\author{
B. Eslam Panah ${ }^{1,2,3, a} \mathbb{i}$, T. Yazdizadeh ${ }^{4,5, b}$, G. H. Bordbar ${ }^{1,6, c}$ \\ ${ }^{1}$ Physics Department and Biruni Observatory, College of Sciences, Shiraz University, Shiraz 71454, Iran \\ ${ }^{2}$ Research Institute for Astronomy and Astrophysics of Maragha (RIAAM), P.O. Box 55134-441, Maragha, Iran \\ ${ }^{3}$ ICRANet, Piazza della Repubblica 10, 65122 Pescara, Italy \\ ${ }^{4}$ Department of Physics, Payame Noor University (PNU), P.O. Box 19395-3697, Tehran, Iran \\ ${ }_{6}^{5}$ Islamic Azad University, Bafgh Branch, Bafgh 89751-43398, Iran \\ ${ }^{6}$ Department of Physics and Astronomy, University of Waterloo, 200 University Avenue West, Waterloo, ON N2L3G1, Canada
}

Received: 28 November 2018 / Accepted: 22 September 2019 / Published online: 4 October 2019

(C) The Author(s) 2019

\begin{abstract}
Motivated by importance of the existence of quark matter on structure of neutron star. For this purpose, we use a suitable equation of state (EoS) which include three different parts: (i) a layer of hadronic matter, (ii) a mixed phase of quarks and hadrons, and, (iii) a strange quark matter in the core. For this system, in order to do more investigation of the EoS, we evaluate energy, Le Chatelier's principle and stability conditions. Our results show that the EoS satisfies these conditions. Considering this EoS, we study the effect of quark matter on the structure of neutron stars such as maximum mass and the corresponding radius, average density, compactness, Kretschmann scalar, Schwarzschild radius, gravitational redshift and dynamical stability. Also, considering the mentioned EoS in this paper, we find that the maximum mass of hybrid stars is a little smaller than that of the corresponding pure neutron star. Indeed the maximum mass of hybrid stars can be quite close to the pure neutron stars. Our calculations about the dynamical stability show that these stars are stable against the radial adiabatic infinitesimal perturbations. In addition, our analyze indicates that neutron stars are under a contraction due to the existence of quark core.
\end{abstract}

\section{Introduction}

Neutron stars which are born in the aftermath of corecollapsing supernova (SN) explosions, are a cosmic laboratory and the best environment for the studying dense matter problems. It is notable that, in the center of neutron star because of high densities, the matter is envisaged to have a

\footnotetext{
${ }^{\text {a } e-m a i l: ~ b e s l a m p a n a h @ s h i r a z u . a c . i r ~}$

be-mail: tyazdizadeh@yahoo.com

c e-mail: ghbordbar@shiraz.ac.ir
}

transition from hadronic matter to strange quark matter, see Refs. [1-4], for more details. Also, Glendenning in Ref. [5], showed that proper construction of phase transition between the hadron and quark, inside the neutron stars implies the coexistence of nucleonic matter and quark matter over a finite range of the pressure. Accordingly, a mixed hadronquark phase exists in the neutron star, so that its energy is lower than that of the quark matter and nucleonic matter. Phase transition between quark matter core and hadronic external layers (hadron-quark phase) in neutron star is an interesting subject which has been investigated by many authors [6-18]. For example, the effects of quark-hadron matter in the center of neutron stars have been studied in Refs. $[19,20]$, and the obtained results have been shown that for PSR J1614 - 2230, and PSR J0348 + 0432 with masses about $2 M_{\odot}$, may contain a region of quark-hybrid matter in their center. Plumari et al. have investigated the effects of a quark core inside neutron star by considering the quarkgluon EoS in the framework of field correlator model [21]. They have found an upper limit for the mass of neutron stars by adjusting some parameters. This limit was in the range $M_{\max } \simeq 2 M_{\odot}$. In addition, Chen et al. have studied cold dense quark matter and hybrid stars with a Dyson-Schwinger quark model and various choices of the quark-gluon vertex [22]. They have showed that hadron states have the maximum mass lower than the pure nucleonic neutron stars, but higher than two solar masses. Their results depended on parameters of EoS. Also, Lastowiecki et al. have found that compact stars masses of about $2 M_{\odot}$ such as PSR J1614-2230 and PSR J3048 + 0432 were compatible with the possible existence of deconfined quark matter in their core [23]. Neutrino emissivity in the quark-hadron mixed phase of neutron stars have been investigated in Ref. [24]. According to 
importance of existence of quark matter in the neutron stars [15,25-30,30-49], we consider a neutron star to be composed of a hadronic matter layer, a mixed phase of quarks and hadrons, and in the core of star, a quark matter. One of our aims in this work is determining the structure of neutron star with a quark core and comparing it with observation data.

In order to study the structure of stars and their phenomenological properties, we must use the hydrostatic equilibrium equation (HEE). Indeed, this equation is based on the fact that a typical star will be in equilibrium when there is a balance between the gravitational force and the internal pressure. The first HEE equation was introduced by Tolman, Oppenheimer and Volkoff (TOV) [50-52] in the Einstein gravity which is known as TOV equation. Considering TOV equation, the structure of compact stars have been evaluated by many authors in Refs. [53-61].

According to this fact that, there are some massive neutron stars with mass more than two times of solar mass, $M \geq$ $2 M_{\odot}$, for example, $4 U 1700-377$ with $M=2.4 M_{\odot}$ [62], and $J 1748-2021 B$ with $M=2.7 M_{\odot}$ [63], one of our goals in this work is related to answer this question: is there a quark core inside neutron stars with mass more than $2 M_{\odot}$ ( $M \geq 2 M_{\odot}$ ), by considering a suitable EoS which obtained by combining three different parts, a layer of hadronic matter, a mixed phase of quarks and hadrons, and a strange quark matter in core? In order to evaluate a suitable EoS, we will study energy, Le Chatelier's principle and stability conditions of this EoS. Another our goal is related to the investigation of the effects of quark core in the structure of hybrid star. For this goal, we study the difference between the structure of hybrid stars and pure neutron stars.

The outline of the paper is as follows; First, in order to investigate the structure of neutron stars, we evaluate a suitable EoS which includes three different layers. Then, we compare the structure of neutron stars by considering the EoS with and without a quark core. Indeed, we study the effects of EoS by applying a quark core on the structure of neutron stars. Next, we compare our obtained results with those of observational data. The last section is devoted to closing remarks.

\section{Equation of state}

A neutron star with a quark core composed of a hadronic matter layer, a mixed part of quarks and hadrons and a quark matter in core. Thus we calculate the EoS of different parts of this star in the following subsections.

\subsection{Hadron phase}

We use the lowest order constrained variational (LOCV) many-body method to determine the EoS of nucleonic mat- ter [64-67]. We consider a cluster expansion of the energy functional up to the two-body term,

$E([f])=\frac{\langle\psi|H| \psi\rangle}{N\langle\psi \mid \psi\rangle}=E_{1}+E_{2}$,

in which $H$ is the Hamiltonian of the system. Also, $\psi$ is the total wave function in which we consider a trail manybody wave function as $\psi=F \phi$. Here $\phi$ is a uncorrelated ground-state wave function of $N$ independent nucleons, and $F$ is a proper $N$-body correlation function which is taken according to the Jastrow ansatz, $F=\mathcal{S} \prod_{i>j} f(i j)$, in which $\mathcal{S}$ and $f(i j)$ are the symmetrizing operator and the two-body correlation function, respectively. Using the Jastrow ansatz and after some algebra, the energy is calculated (see [68], for more details). The one body term is $E_{1}=\sum_{i=1,2} \frac{3}{5} \frac{\hbar^{2} k_{i}^{2}}{2 m_{i}} \frac{\rho_{i}}{\rho}$, for an asymmetrical nucleonic matter, where $\rho_{i}$ are the nucleonic densities associated with the protons and neutrons $\left(\rho=\rho_{p}+\rho_{n}\right)$ and $k_{i}=\left(6 \pi^{2} \rho_{i}\right)^{1 / 3}$ is the Fermi momentum of particle $i$. The two-body energy is $E_{2}=\frac{1}{2 N} \sum_{i j}<i j|v(12)| i j-j i>$. In this relation, the operator $v(12)$ is the effective nuclear potential. The complete calculation for nuclear matter has been presented in Ref. [65]. It should be noted that for calculation of the structure properties of neutron star, the equation of state of hadronic matter has a crucial role. On the other hand, various nucleon-nucleon potentials lead to the different equations of state. Therefore, the maximum mass of neutron star depends highly on the inter-nucleon potential [69]. Here the three-body nucleon-nucleon potential is very important for the nucleonic matter calculations. In the present paper, for the hadronic matter calculations, we use the $U V 14+T N I$ potential in which the effect of three-body nuclear force has been considered.

\subsection{Quark phase}

The total energy of strange quark matter with deconfined up (u), down (d) and strange (s) quarks within MIT bag model $[70,71]$ is given by

$\mathcal{E}_{\text {tot }}=\mathcal{E}_{u}+\mathcal{E}_{d}+\mathcal{E}_{s}+B$

The quark confinement in MIT bag model is satisfied by a density dependent bag constant $B$, that is interpreted as the difference between energy densities of non interacting and interacting quarks. We use a density dependent with the Gaussian form [72,73],

$B(\rho)=B_{\infty}+\left(B_{0}-B_{\infty}\right) \exp \left[-\beta\left(\frac{\rho}{\rho_{0}}\right)^{2}\right]$ 
In the Eq. (2) $\mathcal{E}_{i}$ is

$\mathcal{E}_{i}=\frac{3 m_{i}^{4}}{8 \pi^{2}}\left[x_{i}\left(2 x_{i}^{2}+1\right)\left(\sqrt{1+x_{i}^{2}}\right)-\sinh ^{-1} x_{i}\right]$,

where $x_{i}=\frac{k_{F}^{(i)}}{m_{i}}$ and, $k_{F}^{(i)}=\left(\rho_{i} \pi^{2}\right)^{1 / 3}$ is the fermi momentum of quark $i$. See Ref. [72] for more details.

Now, by using the energy density from Eq. (2), we can obtain the EoS of quark matter in the MIT bag model,

$P(\rho)=\rho \frac{\partial \mathcal{E}}{\partial \rho}-\mathcal{E}$

\subsection{Mixed phase}

The hadron-quark phase happens in the high range of baryon density values. The occupied fraction of space by quark matter smoothly increases from zero where there is no quark to unity when the last nucleons dissolve into the quarks. In this phase, there are a mixture of hadrons, quarks and electrons. According to the Gibss equilibrium condition, the pressures, the temperatures and chemical potentials of both hadron and quark phases are equal [5], $\left(\mu_{p}^{Q}=\mu_{p}^{H}\right)$ and $\left(\mu_{n}^{Q}=\mu_{n}^{H}\right)$ where $\mu_{n}^{H}\left(\mu_{p}^{H}\right)$ and $\mu_{n}^{Q}\left(\mu_{p}^{Q}\right)$ are neutrons (protons) chemical potential in the hadron phase and the quark phase, respectively. It is notable that $\mu_{n}$ and $\mu_{p}$ are given by

$\mu_{n}=\frac{\partial \mathcal{E}}{\partial \rho_{n}}, \quad \mu_{p}=\frac{\partial \mathcal{E}}{\partial \rho_{p}}$.

As the chemical potentials determine the charge densities, the volume fraction occupied by quark matter, $\chi$, can be obtained by the requirement of global charge neutrality. Then the total energy density and baryon density of mixed phase could be determined,

$$
\begin{aligned}
& \chi\left(\frac{2}{3} \rho_{u}-\frac{1}{3} \rho_{d}-\frac{1}{3} \rho_{s}\right)+(1-\chi) \rho_{p}-\rho_{e}=0, \\
& \chi \rho_{Q}+(1-\chi) \rho_{H}=\rho_{B}, \\
& \chi \mathcal{E}_{Q P}+(1-\chi) \mathcal{E}_{H P}=\mathcal{E}_{M P} .
\end{aligned}
$$

Calculations regarding the EoS of mixed phase has been fully discussed in Ref. [72].

At this stage we can determine EoS of neutron star with quark core using the results of proceeding sections. Also we investigate the energy and stability conditions for our results. For this purpose at first, we extract a mathematical form for the EoS presented as a polynomial function in the following form

$$
P=\sum_{i=1}^{7} a_{i} \mathcal{E}^{7-i}
$$

where $a_{i}$ are

$$
\begin{array}{ll}
a_{1}=1.194 \times 10^{-57}, & a_{2}=-0.2467 \times 10^{-40}, \\
a_{3}=2.011 \times 10^{-25}, & a_{4}=-8.123 \times 10^{-10}, \\
a_{5}=1.656 \times 10^{6}, & a_{6}=-1.201 \times 10^{21}, \\
a_{7}=2.915 \times 10^{35} . &
\end{array}
$$

In order to do more investigation of the obtained EoS, we evaluate energy, Le Chatelier's principle and stability conditions as follows.

\subsection{Energy conditions}

It is expected that the energy conditions are satisfied by any EoS of matter. In general, there are four energy conditions. The null energy condition (NEC), weak energy condition (WEC), strong energy condition (SEC) and dominant energy condition (DEC). The two first cases state that for any matter distribution, energy should be non-negative. The weak energy apply to time-like vectors and the null energy condition is for null vectors. The strange energy condition states that the gravitational field is attractive. The last condition means that the energy flow rate of matter is less than the speed of light [74]. These conditions in the center of neutron star are given as follows

$$
\begin{aligned}
N E C & \rightarrow P_{c}+\mathcal{E}_{c} \geq 0, \\
W E C & \rightarrow P_{c}+\mathcal{E}_{c} \geq 0 \quad \& \quad \mathcal{E}_{c} \geq 0, \\
S E C & \rightarrow P_{c}+\mathcal{E}_{c} \geq 0 \quad \& \quad 3 P_{c}+\mathcal{E}_{c} \geq 0, \\
D E C & \rightarrow \mathcal{E}_{c}>\left|P_{c}\right|,
\end{aligned}
$$

where $\mathcal{E}_{c}$ and $P_{c}$ are the density and pressure in the center of neutron star $(r=0)$, respectively. The results of above conditions for our EoS are given in Table 1.

We see that our EoS satisfied all mentioned energy conditions.

\subsection{Stability}

According to the stability condition for the EoS of neutron star matter in a physically acceptable model, the corresponding extracted velocity of sound $(v)$ must be less than the light velocity $(c)[75,76]$. Thus the stability condition is given $0 \leq v^{2}=\left(\frac{d P}{d \mathcal{E}}\right) \leq c^{2}$ or $0 \leq \frac{v^{2}}{c^{2}}=\frac{1}{c^{2}}\left(\frac{d P}{d \mathcal{E}}\right) \leq 1$. Using Eq. 9 we compute $\frac{v^{2}}{c^{2}}$ versus density which is presented in Fig. 1. It is evident that stability condition is satisfied by the EoS of neutron star with quark matter.

\subsection{Le Chatelier's principle}

Le Chatelier's principle is defined as: the matter of star satisfies $d P / d \mathcal{E} \geq 0$ which is a essential condition of a stable body both as a whole and also with respect to the non- 
Table 1 Energy conditions for neutron star with a quark core

\begin{tabular}{lllllll}
\hline Type of star & $\mathcal{E}_{c}\left(10^{14} \mathrm{~g} / \mathrm{cm}^{3}\right)$ & $P_{c}\left(10^{14} \mathrm{~g} / \mathrm{cm}^{3}\right)$ & NEC & WEC & SEC & DEC \\
\hline NS + QuarkCore & 25.8 & 7.8 & $\sqrt{ }$ & $\sqrt{ }$ & $\sqrt{ }$ & $\sqrt{ }$ \\
\hline
\end{tabular}

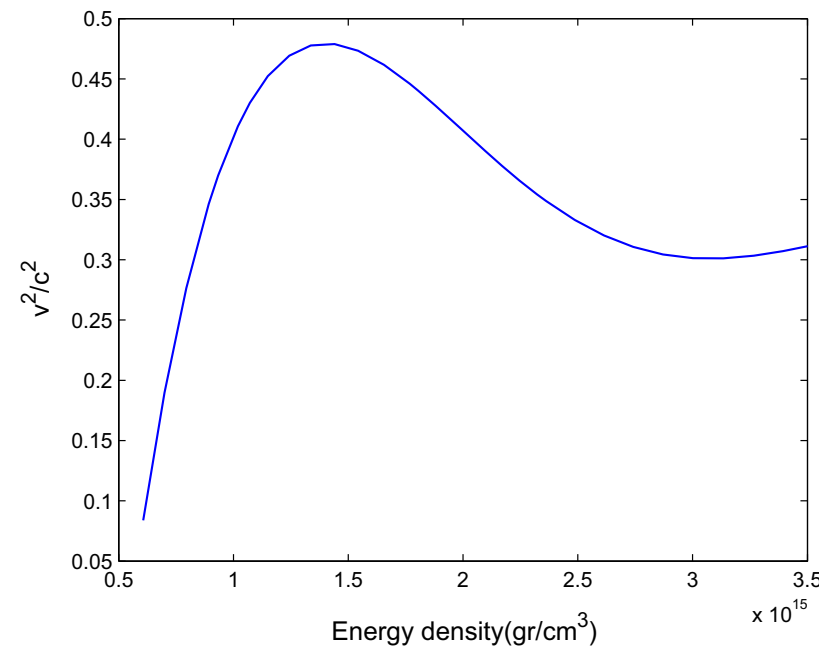

Fig. 1 Sound speed versus density

equilibrium elementary regions with spontaneous expansion or contraction [77]. According the Fig. 1, the Le Chatelier's principle is established.

The above results show that we encounter with a suitable EoS for studying the neutron stars with a quark matter in the core. Therefore, we consider our EoS and investigate the structure of neutron star with three different layers.

\section{Structure of the neutron star with and without quark matter}

In this section, we study the effect of quark matter on the structure of neutron stars, and then compare our results with the structure of neutron stars without quark matter. Employing the obtained EoS for neutron star with the quark matter and using TOV equation, we have gotten interesting results which are given in Table 2.

As one can see, considering the quark matter in the core of neutron stars change their structure properties. Indeed, there are some interesting results when we consider the quark matter in the calculation of structure of neutron stars. For exam-

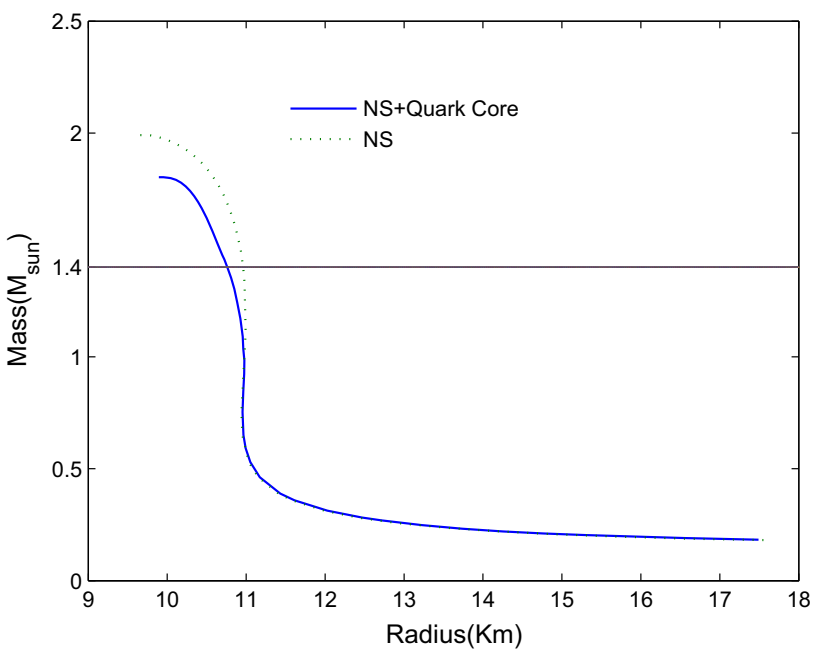

Fig. 2 Mass-radius relation for the neutron stars with and without the quark matter

ple, by considering the quark matter, the maximum mass decreases (see Table 2 and Fig. 2, for more details). In order to do more investigation of the effect of quark matter, we calculate another properties of the neutron star such as the average density, compactness, Kretschmann scalar, gravitational redshift and dynamical stability.

\subsection{Average density}

The average density of an object has the following form,

$\bar{\rho}=\frac{M}{\frac{4}{3} \pi R^{3}}$,

where for our system, $M$ and $R$ are the gravitational mass and radius of a neutron star. The presented results in Table 2, show that by considering the quark matter in the neutron star core, the average density from the perspective of a distant observer (or a observer outside the neutron star) decreases. Indeed, by adding the quark matter to the core of neutron star, the total average density (this average density is not related to density of center of neutron star) of this system decreases.

Table 2 Structure properties of neutron star without quark matter (NS) and with quark core (NS + Q)

\begin{tabular}{lllllllll}
\hline Type of star & $M_{\max }\left(M_{\odot}\right)$ & $R(\mathrm{~km})$ & $\mathcal{E}_{c}\left(10^{14} \mathrm{~g} / \mathrm{cm}^{3}\right)$ & $R_{S c h}(\mathrm{~km})$ & $\bar{\rho}\left(10^{14} \mathrm{~g} / \mathrm{cm}^{3}\right)$ & $\sigma$ & $K\left(10^{-8} \mathrm{~m}^{-2}\right)$ & $z$ \\
\hline NS & 1.98 & 9.8 & 27.1 & 5.84 & 9.99 & 0.59 & 2.15 \\
NS + Quark Core & 1.8 & 10 & 25.8 & 5.31 & 8.55 & 0.53 & 1.84 \\
\hline
\end{tabular}




\subsection{Compactness}

The compactness of a spherical object is usually defined as the ratio of Schwarzschild radius to the radius of object $\left(\sigma=\frac{R_{S c h}}{R}\right)$, which may be indicated as the strength of gravity of compact objects. Our results for the compactness are presented in Table 2. Similar to the average density, by applying the quark matter in the calculation of structure of neutron star, the compactness from the perspective of a distant observer (or a observer outside the neutron star) decreases.

\subsection{Kretschmann scalar}

Another quantity that gives us information of the strength of gravity is related to the spacetime curvature. According to this fact that, in the Schwarzschild spacetime, the components of the Ricci tensor $\left(R_{\mu \nu}\right)$ and the Ricci scalar $\left(R_{\mu \nu} R^{\mu v}\right)$ are zero outside the star, and therefore these quantities do not give us any information about the spacetime curvature. Therefore, we use another quantity in order to evaluate the curvature of spacetime. The quantity that can help us to find out the curvature of spacetime is related to the Riemann tensor $\left(R_{\mu \nu \gamma \delta}\right)$. The Riemann tensor may have more components, and also for simplicity, we can evaluate the Kretschmann scalar for measurement of the curvature in a vacuum. So, the curvature at the surface of a neutron star is given as [78-80]

$K=\frac{4 \sqrt{3} G M}{c^{2} R^{3}}$.

Our results confirm that by adding the quark matter to the core of neutron star, the strength of gravity decreases (see Table 2).

\subsection{Gravitational redshift}

The gravitational redshift is given as follows,

$$
z=\frac{1}{\sqrt{1-\frac{2 G M}{c^{2} R}}}-1 .
$$

The result related to the gravitational redshift are given in Table 2. This result shows that by considering a quark core inside a neutron star, the gravitational redshift decreases.

\subsection{Dynamical stability}

Here we use Shapiro and Teukolsky's approach [81], for studying the dynamical stability of stellar model against the infinitesimal radial adiabatic perturbation. In this approach, the dynamical stability condition only require that the pressure-averaged value of the adiabatic index be more than
$4 / 3$, but not for the local value of the adiabatic index. This relation is given by

$\bar{\gamma}=\frac{\int_{0}^{R} \gamma p r^{2} d r}{\int_{0}^{R} p r^{2} d r}$,

where it must be more than $4 / 3$. Here, we consider Shapiro and Teukolsky's approach for evaluating the dynamical stability of hybrid stars extracted from our EoS. Our calculation about the pressure-averaged value of the adiabatic index (Eq. 18) are

$$
\begin{aligned}
\bar{\gamma}_{N S} & =2.98, \\
\bar{\gamma}_{N S+Q} & =2.38 .
\end{aligned}
$$

The above quantities show that the pressure-averaged value of the adiabatic index for neutron stars with and without a quark core are 2.38 and 2.98 , respectively. Therefore these quantities are more than $4 / 3$. This shows that the obtained pure neutron stars and hybrid stars obey the dynamical stability.

Another interesting results is related to contraction of neutron stars due to the quark core (see Tables 3 and 4, for more details). In the other words, the obtained results in Tables 3 and 4 and Figs. 2 and 3 show that the radius of neutron stars without the quark matter (NS) and with the gravitational mass equal to $1.4 M_{\odot}$ (or $1.8 M_{\odot}$ ) are greater than the radius of neutron stars with a quark core $(\mathrm{NS}+\mathrm{Q})$. Indeed, for the same gravitational masses of NS and NS + Q, the compactness, the Kretschmann scalar and the gravitational redshift increase due to the reduced radius. In addition, this difference appears for the neutron stars with the gravitational mass higher than the solar mass $\left(M \geq M_{\odot}\right)$ (see Figs. 2 and $3)$.

Briefly, we can see that the existence of quark matter inside the neutron stars leads to decreasing for the maximum mass and so it contracts them. These results indicate that by applying the mentioned EoS in Eq. (9), the cores of neutron stars

Table 3 Properties of neutron star with the gravitational mass equal to $1.4 M_{\odot}$ for NS and NS + Q

\begin{tabular}{lllll}
\hline Type of star & $R(\mathrm{~km})$ & $\sigma$ & $K\left(10^{-8} \mathrm{~m}^{-2}\right)$ & $z$ \\
\hline NS & 10.96 & 0.37 & 1.09 & 0.26 \\
NS + Quark Core & 10.76 & 0.38 & 1.15 & 0.27 \\
\hline
\end{tabular}

Table 4 Properties of neutron star with the gravitational mass equal to $1.8 M_{\odot}$ for NS and NS+Q

\begin{tabular}{lllll}
\hline Type of star & $R(\mathrm{~km})$ & $\sigma$ & $K\left(10^{-8} \mathrm{~m}^{-2}\right)$ & $z$ \\
\hline NS & 10.56 & 0.50 & 1.56 & 0.42 \\
NS + Quark Core & 10.00 & 0.53 & 1.84 & 0.46 \\
\hline
\end{tabular}




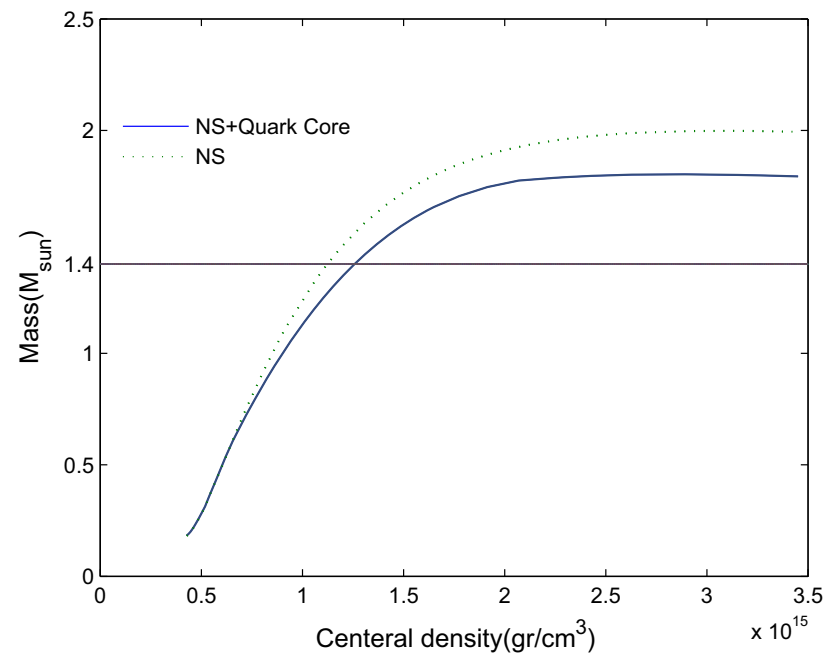

Fig. 3 Mass-the central density relation for the neutron stars with and without the quark matter

with mass more than $2 M_{\odot}$, do not have any quark matter. Indeed, the presented EoS in paper (Eq. 9), can not predict the existence of massive hybrid stars with more than $2 M_{\odot}$, because by adding the quark matter to the structure of these stars, the maximum masses decreases.

\section{Summary and conclusion}

The paper at hand studied the structure of cold hybrid stars which included three different parts: (i) a layer of hadronic matter, (ii) a mixed phase of quarks and hadrons, and (iii) a quark matter in the core. For layer of hadronic matter we used the lowest-order constrained variational (LOCV) manybody method employing the $U V 14+T N I$ potential for the nucleon-nucleon interaction (for more details about neutron star with hadron matter and $U V 14+T N I$ potential see Ref. [69]). In another layer (mixed phase of quarks and hadrons), we considered Gibss equilibrium condition. Indeed for this layer, the temperature, pressures and chemical potentials of both hadron and quark phases are equal. Finally, we considered a quark matter in the core of neutron stars, and for this region, we used the total energy which included up, down and strange quarks within MIT bag model. We applied TOV equation for obtaining the structure properties of these stars. Our results indicated that the maximum mass, the average density, compactness, gravitational redshift, and Kretschmann scalar of neutron stars decrease by adding a quark core to them. These results led to contraction of hybrid stars. On the other hand, by applying the mentioned EoS in this paper, we found an upper limit for the maximum mass of hybrid stars. In other word, hybrid stars in our model, did not have mass more than two times of the solar mass $\left(M_{\max } \leq 2 M_{\odot}\right)$. It is notable that, Hoyos et al. have evaluated the properties of neutron stars by applying top-down holographic model for strongly interacting quark matter [82]. They have obtained an EoS which was matched with state-of-the-art results for dense nuclear. Also, they have solved TOV equation with their EoS, and found the maximal stellar masses in the excess of two solar masses. Their results showed that there are no any quark matter inside these massive neutron stars. In other words, their results confirm our conclusions about the existence an upper limit for neutron stars with a quark core, i.e, $M_{\max } \leq 2 M_{\odot}$. However there were some difference between their results with ours. For example, the obtained radius of neutron stars in our calculations were greater than their results (the radius of a

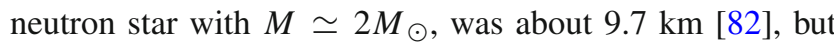
we found that it was about $10 \mathrm{~km}$ ). Finally, we investigated the dynamical stability of neutron stars with and without the quark core. Our calculations indicated that these neutron stars are stable against the radial adiabatic infinitesimal perturbations. Briefly, we obtained the quite interesting results for hybrid stars, such as;

(i) the EoS derived in this work satisfied the energy, Le Chatelier and stability conditions.

(ii) considering the EoS introduced in this paper, we found that the maximum mass of cold hybrid stars could not be more than $2 M_{\odot}\left(M_{\max } \leq 2 M_{\odot}\right)$. In other word, there are no cold massive hybrid stars in the mass range $M_{\max }>$ $2 M_{\odot}$, when we applied our EoS. Therefore, our results showed that inside the neutron stars such as $4 U 1700-$ 377 [62] with the mass about $2.4 M_{\odot}$, and $J 1748-2021 B$ [63] with the mass about $2.7 M_{\odot}$, there is no any quark matter.

(iii) the maximum mass, the average density, compactness, the Kretschmann scalar, and gravitational redshift of neutron stars decrease owing to the existence of quark matter in the structure them.

(iv) the neutron stars are contracted due to the presence of quark matter in their center.

(v) the obtained results indicated that the studied hybrid stars in this paper are stable against the radial adiabatic infinitesimal perturbations.

(vi) for neutron stars with the gravitational mass more than one solar mass $\left(M \geq M_{\odot}\right)$, there was a difference between NS and NS + Q. In other words, there was no any difference between the properties of NS and NS + Q in the range $M<M_{\odot}$.

It will be worthwhile to study the effects of the magnetic field [83-86], generalization of static compact objects to anisotropic [87-92], rotating [93-102], rapidly rotating [103-110] on the structure properties of neutron stars with a quark matter in core can be interesting topics. We leave these issues for future works. 
Acknowledgements We thank an anonymous referee for useful comments. We wish to thank Shiraz University Research Council. This work has been supported financially by the Research Institute for Astronomy and Astrophysics of Maragha (RIAAM) under research project No. 1/5750-56. TY wishes to thank the Research Council of Islamic Azad University, Bafgh Branch.

Data Availability Statement This manuscript has no associated data or the data will not be deposited. [Authors' comment: This is a theoretical study and no experimental data has been listed.]

Open Access This article is distributed under the terms of the Creative Commons Attribution 4.0 International License (http://creativecomm ons.org/licenses/by/4.0/), which permits unrestricted use, distribution, and reproduction in any medium, provided you give appropriate credit to the original author(s) and the source, provide a link to the Creative Commons license, and indicate if changes were made. Funded by SCOAP ${ }^{3}$.

\section{References}

1. D.D. Ivanenko, D.F. Kurdgelaidze, Astrophysics 1, 251 (1965)

2. N. Itoh, Progr. Theor. Phys. 44, 291 (1970)

3. H. Fritzsch, M. Gell-Mann, H. Leutwyler, Phys. Lett. B 47, 365 (1973)

4. J.C. Collins, M.J. Perry, Phys. Rev. Lett. 34, 1353 (1975)

5. N.K. Glendenning, Phys. Rev. D 46, 1274 (1992)

6. S.K. Ghosh, S.C. Phatak, P.K. Sahu, Z. Phys. A 352, 457 (1995)

7. S. Kubis, M. Kutschera, Phys. Rev. Lett. 76, 3876 (1996)

8. D. Bandyopadhyay, S. Chakrabarty, S. Pal, Phys. Rev. Lett. 79, 2176 (1997)

9. A. Steiner, M. Prakash, J.M. Lattimer, Phys. Lett. B 486, 239 (2000)

10. G.F. Burgio, M. Baldo, H.-J. Schulze, P.K. Sahu, Phys. Rev. C 66, $025802(2002)$

11. G. Lugones, T.A.S. do Carmo, A.G. Grunfeld, N.N. Scoccola, Phys. Rev. D 81, 085012 (2010)

12. N. Yasutake, T. Maruyama, T. Tatsumi, Phys. Rev. D 86, 101302 (2012)

13. C.H. Lenzi, G. Lugones, Astrophys. J. 759, 57 (2012)

14. N. Chamel, A.F. Fantina, J.M. Pearson, S. Goriely, Proc. Int. Astron. Union 8, 356 (2012)

15. B. Franzon, R.O. Gomes, S. Schramm, Mon. Not. R. Astron. Soc. 463, 571 (2016)

16. J.P. Pereira, C.V. Flores, G. Lugones, Astrophys. J. 860, 12 (2018)

17. E.R. Most, L.J. Papenfort, V. Dexheimer, M. Hanauske, S. Schramm, H. Stcker, L. Rezzolla, Phys. Rev. Lett. 122, 061101 (2019)

18. J.P. Pereira, G. Lugones, Astrophys. J. 871, 47 (2019)

19. M. Orsaria, H. Rodrigues, F. Weber, G.A. Contrera, Phys. Rev. D 87, 023001 (2013)

20. M. Orsaria, H. Rodrigues, F. Weber, G.A. Contrera, Phys. Rev. C 89, 015806 (2014)

21. S. Plumari, G.F. Burgio, V. Greco, D. Zappala, Phys. Rev. D 88, 083005 (2013)

22. H. Chen, J.-B. Wei, M. Baldo, G.F. Burgio, H.-J. Schulze, Phys. Rev. D 91, 105002 (2015)

23. R. Lastowiecki, D. Blaschke, T. Fischer, T. Klahn, Phys. Part. Nucl. 46, 843 (2015)

24. W.M. Spinella, F. Weber, G.A. Contrera, M.G. Orsaria, Eur. Phys. J. A 52, 61 (2016)

25. H. Mishra, S.P. Misra, P.K. Panda, B.K. Parida, Int. J. Mod. Phys. E 2, 547 (1993)

26. M.L. Olesen, J. Madsen, Phys. Rev. D 49, 2698 (1994)
27. D. Bandyopadhyay, S. Chakrabarty, S. Pal, Phys. Rev. Lett. 79, 2176 (1997)

28. M.B. Christiansen, N.K. Glendenning, Phys. Rev. C 56, 2858 (1997)

29. S. Pal, M. Hanauske, I. Zakout, H. Stocker, W. Greiner, Phys. Rev. C 60, 015802 (1999)

30. A.W. Steiner, M. Prakash, J.M. Lattimer, Phys. Lett. B 486, 239 (2000)

31. R. Oechslin, G. Poghosyan, K. Uryu, Nucl. Phys. A 718, 706 (2003)

32. T. Harko, K.S. Cheng, P.S. Tang, Astrophys. J. 608, 945 (2004)

33. J. Staff, R. Ouyed, P. Jaikumar, Astrophys. J. 645, L145 (2006)

34. P. Jaikumar, S.W. Reddy, A.W. Steiner, Mod. Phys. Lett. A 21, 1965 (2006)

35. I. Bombaci, G. Lugones, I. Vidana, Astron. Astrophys. 462, 1017 (2007)

36. F. Yang, H. Shen, Phys. Rev. C 77, 025801 (2008)

37. M.G. Alford, Nucl. Phys. A 830, 385c (2009)

38. G. Rupak, P. Jaikumar, Phys. Rev. C 82, 055806 (2010)

39. I. Bombaci, D. Logoteta, C. Providencia, I. Vidana, Astron. Astrophys. 528, A71 (2011)

40. G.Y. Shao, Phys. Lett. B 704, 343 (2011)

41. N. Yasutake, G.F. Burgio, H.J. Schulze, Phys. Atom. Nucl. 74, 1502 (2011)

42. D. Logoteta, I. Bombaci, C. Providencia, I. Vidana, Phys. Rev. D 85, 023003 (2012)

43. G.Y. Shao, M. Colonna, M. Di Toro, Y.X. Liu, B. Liu, Phys. Rev. D 87, 096012 (2013)

44. B. Franzon, V. Dexheimer, S. Schramm, Mon. Not. R. Astron. Soc. 456, 2937 (2015)

45. S.M. de Carvalho et al., Phys. Rev. C 92, 035810 (2015)

46. T. Miyatsu, M.-K. Cheoun, K. Saito, Astrophys. J. 813, 135 (2015)

47. I. Bombaci, D. Logoteta, I. Vidana, C. Providencia, Eur. Phys. J. A 52, 58 (2016)

48. A. Mukherjee, S. Schramm, J. Steinheimer, V. Dexheimer, Astron. Astrophys. 608, A110 (2017)

49. G. Baym, T. Hatsuda, T. Kojo, P.D. Powell, Y. Song, T. Takatsuka, Rep. Progr. Phys. 81, 056902 (2018)

50. R.C. Tolman, Proc. Natl. Acad. Sci. 20, 169 (1934)

51. R.C. Tolman, Phys. Rev. 55, 364 (1939)

52. J.R. Oppenheimer, G.M. Volkoff, Phys. Rev. 55, 374 (1939)

53. N.K. Glendenning, Compact Star, Nuclear Physics, Particle Physics, and General Relativity (Springer, New York, 2000)

54. F. Weber, Pulsars as Astrophysical Laboratories for Nuclear and Particle Physics (Institute of Physics, Bristol, 1999)

55. N. Yunes, M. Visser, Int. J. Mod. Phys. A 18, 3433 (2003)

56. R.R. Silbar, S. Reddy, Am. J. Phys. 72, 892 (2004)

57. G. Narain, J. Schaffner-Bielich, I.N. Mishustin, Phys. Rev. D 74, 063003 (2006)

58. P. Boonserm, M. Visser, S. Weinfurtner, Phys. Rev. D 76, 044024 (2007)

59. X. Li, F. Wang, K.S. Cheng, J. Cosmol. Astropart. Phys. 10, 031 (2012)

60. A.M. Oliveira, H.E.S. Velten, J.C. Fabris, I.G. Salako, Eur. Phys. J. C 74, 3170 (2014)

61. X.T. He, F.J. Fattoyev, B.A. Li, W.G. Newton, Phys. Rev. C 91, $015810(2015)$

62. J.S. Clark et al., Astron. Astrophys. 392, 909 (2002)

63. P.C.C. Freire et al., Astrophys. J. 675, 670 (2008)

64. G.H. Bordbar, M. Modarres, J. Phys. G Nucl. Part. Phys. 23, 1631 (1997)

65. G.H. Bordbar, M. Modarres, Phys. Rev. C 57, 714 (1998)

66. M. Modarres, G.H. Bordbar, Phys. Rev. C 58, 2781 (1998)

67. M. Bigdeli, G.H. Bordbar, Z. Rezaei, Phys. Rev. C 80, 034310 (2009)

68. J.W. Clark, Prog. Part. Nucl. Phys. 2, 89 (1979) 
69. G.H. Bordbar, M. Hayati, Int. J. Mod. Phys. A 21, 1555 (2006)

70. A. Chodos, R.L. Jaffe, K. Johnson, C.B. Thorn, V.F. Weisskopf, Phys. Rev. D 9, 3471 (1974)

71. G. Baym, E.W. Kolb, L. McLerran, T.P. Walker, R.L. Jaffe, Phys. Lett. B 160, 181 (1985)

72. G.H. Bordbar, M. Bigdeli, T. Yazdizade, Int. J. Mod. Phys. A 21, 5991 (2006)

73. G.F. Burgio, M. Baldo, P.K. Sahu, H.J. Schulze, Phys. Rev. C 66, $025802(2002)$

74. E. Poisson, A Relativist's Toolkit (Cambrige University Press, New York, 2004)

75. L. Herrera, Phys. Lett. A 165, 206 (1992)

76. H. Abreu, H. Hernandez, L.A. Nunez, Class. Quantum Gravity 24, 4631 (2007)

77. N.K. Glendenning, Phys. Rev. Lett. 85, 1150 (2000)

78. B. Eslam Panah et al., Astrophys. J. 848, 24 (2017)

79. D. Psaltis, Living Rev. Relativ. 11, 9 (2008)

80. K.Y. Eksi, C. Gungor, M.M. Turkoglu, Phys. Rev. D 89, 063003 (2014)

81. S.L. Shapiro, S.A. Teukolsky, Black Holes, White Dwarfs and Neutron Stars: The Physics of Compact Objects (Wiley, New York, 1983)

82. C. Hoyos, D.R. Fernandez, N. Jokela, A. Vuorinen, Phys. Rev. Lett. 117, 032501 (2016)

83. M. Bocquet, S. Bonazzola, E. Gourgoulhon, J. Novak, Astron. Astrophys. 301, 757 (1995)

84. J.S. Heyl, S. Kulkarni, Astrophys. J. Lett. 506, L61 (1998)

85. C.Y. Cardall, M. Prakash, J.M. Lattimer, Astrophys. J. 554, 322 (2001)

86. D. Chatterjee, T. Elghozi, J. Novak, M. Oertel, Mon. Not. R. Astron. Soc. 447, 3785 (2015)

87. J.A. Miralles, J.A. Pons, V. Urpin, Astron. Astrophys. 420, 245 (2004)

88. C.G. Boehmer, T. Harko, Class. Quantum Gravity 23, 6479 (2006)

89. B.C. Paul, R. Deb, Astrophys. Space Sci. 354, 421 (2014)

90. S.K. Maurya, Y.K. Gupta, S. Ray, B. Dayanandan, Eur. Phys. J. C 75, 225 (2015)

91. D.K. Matondo, S.D. Maharaj, Astrophys. Space Sci. 361, 221 (2016)
92. G. Estevez-Delgado, J. Estevez-Delgado, Eur. Phys. J. C 78, 673 (2018)

93. H. Heiselberg, M. Hjorth-Jensen, Phys. Rev. Lett. 80, 5485 (1998)

94. E. Chubarian, H. Grigorian, G.S. Poghosyan, D. Blaschke, Astron. Astrophys. 357, 968 (2000)

95. N. Andersson, G.L. Comer, Class. Quantum Gravity 18, 969 (2001)

96. Z.B. Etienne, Y.T. Liu, S.L. Shapiro, Phys. Rev. D 74, 044030 (2006)

97. J. Zdunik, M. Bejger, P. Haensel, E. Gourgoulhon, Astron. Astrophys. 450, 747 (2006)

98. P. Pani, E. Berti, Phys. Rev. D 90, 024025 (2014)

99. R.F.P. Mendes, G.E.A. Matsas, D.A.T. Vanzella, Phys. Rev. D 90, 044053 (2014)

100. A. Cisterna, T. Delsate, L. Ducobu, M. Rinaldi, Phys. Rev. D 93, 084046 (2016)

101. J.G. Coelho et al., Astron. Astrophys. 599, A87 (2017)

102. K.V. Staykov, D. Popchev, D.D. Doneva, S.S. Yazadjiev, Eur. Phys. J. C 78, 586 (2018)

103. D. Lai, S.L. Shapiro, Astrophys. J. 442, 259 (1995)

104. S. Yoshida, S. Karino, S. Yoshida, Y. Eriguchi, Mon. Not. R. Astron. Soc. 316, L1 (2000)

105. J. Zdunik, P. Haensel, E. Gourgoulhon, M. Bejger, Astron. Astrophys. 416, 1013 (2004)

106. J. Zdunik, M. Bejger, P. Haensel, E. Gourgoulhon, Astron. Astrophys. 479, 515 (2008)

107. P. Haensel, J. Zdunik, M. Bejger, J. Lattimer, Astron. Astrophys. 502, 605 (2009)

108. D.D. Doneva, S.S. Yazadjiev, N. Stergioulas, K.D. Kokkotas, Phys. Rev. D 88, 084060 (2013)

109. B. Kleihaus, J. Kunz, S. Mojica, M. Zagermann, Phys. Rev. D 93, 064077 (2016)

110. S.-S. Luk, L.-M. Lin, Astrophys. J. 861, 141 (2018) 